
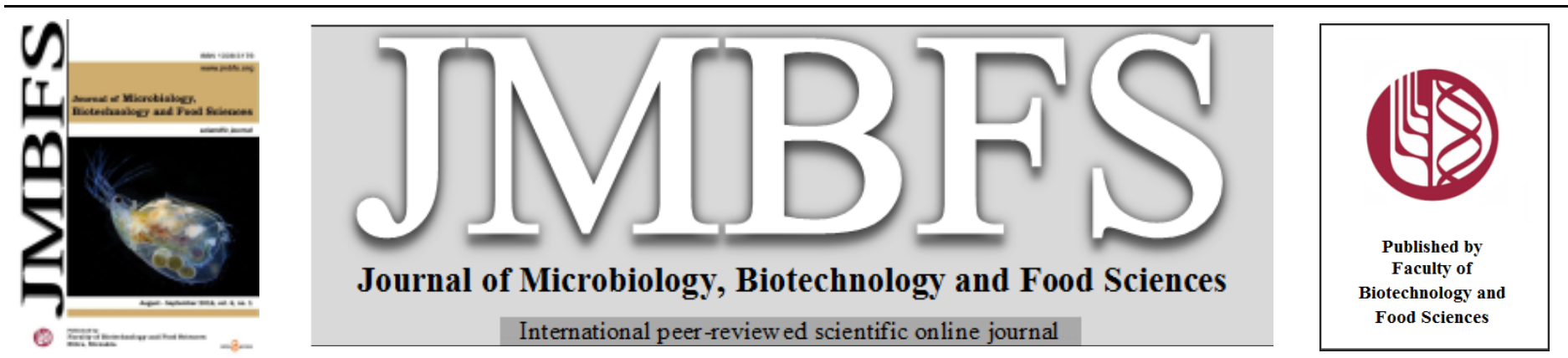

\title{
RAPID AND SIMPLE DETERMINATION OF MINIMUM BIOFILM ERADICATION CONCENTRATION BY A COLORIMETRIC MICROBIAL VIABILITY ASSAY BASED ON REDUCTION OF A WATER-TETRAZOLIUM SALT AND COMBINATED EFFECT OF ANTIBIOTICS AGAINST MICROBIAL BIOFILM
}

\author{
Tadayuki Tsukatani ${ }^{1}$, Tomoaki Kawaguchi ${ }^{1}$, Hikaru Suenaga ${ }^{1}$, Masanobu Shiga ${ }^{2}$ \& Takashi Ikegami ${ }^{2}$
}

Address(es): Dr. Tadayuki Tsukatani,

${ }^{1}$ Biotechnology and Food Research Institute, Fukuoka Industrial Technology Center, Kurume, Japan.

${ }^{2}$ Dojindo Laboratories, Kumamoto, Japan.

*Corresponding author: tukatani@ fitc.pref.fukuoka.jp

doi: 10.15414/jmbfs.2016.6.1.677-680

ARTICLE INFO

Received 7. 8. 2015

Revised 11.3. 2016

Accepted 4. 4. 2016

Published 1. 8. 2016

Regular article

open 2 access

\begin{abstract}
Rapid and simple method for the determination of minimum biofilm eradication concentration (MBEC) using a colorimetric microbial viability assay based on reduction of a tetrazolium salt WST-8 and the biofilm formation method on 96-pegs on the lid of a microtiter plate was developed. The biofilms formed on the 96 pegs were challenged by antibiotics, and the MBEC was then determined from the microbial viability of the biofilms formed on the 96 pegs, assessed by the WST- 8 colorimetric assay. The MBECs obtained by the proposed and conventional methods favorably agreed. The most effective inhibitors of $S$. aureus and $P$. aeruginosa biofilms were vancomycin and ciprofloxacin, respectively. In addition, we clarified that Staphylococcus aureus biofilm was maximally suppressed by a combination of vancomycin, daptomycin, and teicoplanin. The proposed method yields similar performance to conventional methods, but is faster and more easily implemented. Therefore, the proposed method alleviates the tediousness and time-consuming nature of conventional biofilm susceptibility assay.
\end{abstract}

Keywords: Biofilm, minimum biofilm eradication concentration, susceptibility testing, antibiotics, tetrazolium salt

\section{INTRODUCTION}

Biofilms are formed when microorganisms aggregate with each other or adhere to a solid surface and then encase themselves in a self-produced matrix of extracellular polysaccharides and proteins. Once formed, biofilms are extremely difficult to eradicate and their cellular constituents develop increased antimicrobial resistance. Biofilm formation is thought to be directly attributable to various chronic or device-related infections (Bhinu 2005; Bridiera et al. 2011; Burmolle et al., 2010). Therefore, rapid antimicrobial susceptibility testing of microbial biofilm is increasingly important for appropriate patient management and clinical surveillance.

Biofilm-associated infections are treated with different concentrations of antibiotics. To evaluate biofilm resistance to various antibiotics, the biofilm minimum inhibitory concentrations (MICs), broth recovery-based biofilm minimum bactericidal concentrations (MBCs), and minimum biofilm eradication concentrations (MBECs) of the biofilms are assayed (Qu et al., 2010). The biofilm MIC is the concentration that inhibits the immediate release of planktonic microbes from biofilms, and is administered during the acute early stage of biofilm-related infections (Ceri et al., 2001). The biofilm MBC is the concentration that kills $99.9 \%$ of the bacterial cells in the biofilm or sterilizes most of them, and targets chronic biofilm infections (Aaron et al., 2002; Zhang and Mah, 2008). The MBEC is the concentration that definitely eradicates all cells in the biofilm, and is administered for successful treatment of biofilmrelated infections (Ceri et al., 2001). However, a general procedure for the determination of these biofilm parameters is extremely complicated because it is difficult to wash the biofilms cultivated directly on the bottom of wells in microtiter plates and to collect the supernatant on the biofilms with pipetting (Nett et al., 2011; Qu et al., 2010). In addition, this method is prone to contamination.

Alternatively, biofilms can be formed on the 96 pegs on the lid of a microtiter plate (Harrison et al., 2005, 2010). From an evaluation perspective, this approach offers several advantages over the conventional cultivation technique. First, the 96 peg lids coated with biofilms are easily shifted from one microtiter plate to another, enabling an easier wash process. Second, this method avoids accidental disruption of the biofilm by pipetting. However, to determine the MBEC, we need to count the number of bacteria in the established biofilms. For this purpose, we must detach the bacteria from the biofilms established on the 96 pegs and incubate them on agar medium for $24 \mathrm{~h}$

Previously, we developed a method for rapid susceptibility testing based on the reduction of a tetrazolium salt (2-(2-methoxy-4-nitrophenyl)-3-(4-nitrophenyl)-5(2,4-disulfophenyl)-2H-tetrazolium, or monosodium salt (WST-8)) as an indicator reagent (Tsukatani et al., 2009, 2012). In this method, the microorganisms reduce the electron mediator 2-methyl-1,4-naphthoquinone (NQ) to naphthohydroquinone, which in turn reduces WST-8 to formazan. The fina reduction product, formazan, maximally absorbs at $460 \mathrm{~nm}$.

This study aims to develop a rapid and simple method for the determination of the MBEC. In our colorimetric microbial viability assay, biofilms are formed on 96 pegs on the lid of a microtiter plate, and the MBEC is evaluated from the reduction of WST-8 to formazan. The advantages of the proposed method are demonstrated by comparison with conventional methods. The proposed method provides a rapid and simple determination of the MBEC. In addition, we also applied our approach to the biofilm susceptibility of Staphylococcus aureus and Pseudomonas aeruginosa exposed to plural antibiotics.

\section{MATERIAL AND METHODS}

\section{Biofilm growth}

$S$. aureus and $P$. aeruginosa used in this study were obtained from the Biological Resource Center at the National Institute of Technology and Evaluation (NBRC Chiba, Japan), and the American Type Culture Collection (ATCC, Rockville MD, USA). For $P$. aeruginosa, $180 \mu \mathrm{l}$ of bacterial inoculum grown to approximately $10^{6} \mathrm{CFU} \mathrm{m}^{-1}$ in Mueller-Hinton broth (MHB) was added to each well of a 96-well microtiter plate. The plate was then covered with a 96 peg lid and incubated for $24 \mathrm{~h}$, during which time biofilms formed on the 96 pegs Polypropylene pegs (96 pin plate; Stem Co., Tokyo, Japan) was used for $P$. aeruginosa biofilm. For $S$. aureus, $180 \mu \mathrm{l}$ of bacterial inoculum grown to approximately $10^{7} \mathrm{CFU} \mathrm{ml}{ }^{-1}$ in $\mathrm{MHB}$ was added to each well of a 96-well microtiter plate. The plate was then covered with a 96 peg lid and incubated for $72 \mathrm{~h}$. In the experiments involving 72-h biofilms, the 96 peg lids were first incubated for $24 \mathrm{~h}$, then transferred to 96 -well microtiter plates containing fresh $\mathrm{MHB}$, and incubated for another $48 \mathrm{~h}$. Polystyrene pegs (Nunc-Immuno TSP Thermo Fisher Scientific Inc., MA, USA) was used for $S$. aureus biofilm. The 
amounts of established biofilm were measured by a crystal violet stain method (Antunes et al., 2010).

\section{Detection reagents}

The mixture of tetrazolium salt WST-8 (Dojindo Laboratories, Kumamoto, Japan) and 2-methyl-1,4-NQ (Sigma Chemicals, St. Louis, MO, USA) was used as the detection reagent. The WST-8 contains sulfonate groups giving them a net negative charge that reduces their ability to move across cell membranes. Thus, it is necessary to employ 2-methyl-1,4-NQ as an electron mediator to facilitate the cellular reduction of tetrazolium salts. WST-8 was dissolved in distilled water at a concentration of $11.1 \mathrm{mM}$ and the solution was sterilized by passing it through a cellulose acetate membrane filter (pore size $=0.2 \mu \mathrm{m}$ ). 2-Methyl-1,4-NQ (Sigma Chemicals, St. Louis, MO, USA) was dissolved in dimethyl sulfoxide to a concentration of $1.0 \mathrm{mM}$, then mixed with WST-8 solution at a ratio of $1: 9$. The prepared detection reagent contained $10 \mathrm{mM}$ WST-8 and $0.1 \mathrm{mM}$ 2-methyl-1,4NQ.

\section{Determination of MBEC}

The MBEC was determined as shown in Fig. 1. The 96 peg lids with established biofilms were placed onto 96-well microtiter plates containing sterile PBS and vortexed for $30 \mathrm{~s}$ to dislodge any unattached bacteria. After washing three times in sterile PBS, the 96 peg lids were placed onto 96-well microtiter plates containing $180 \mu \mathrm{l}$ of 2-fold serial dilutions of antibiotics (range 1280 to $1 \mu \mathrm{g} \mathrm{ml}^{-1}$ ) in Mueller-Hinton broth (MHB). After $20 \mathrm{~h}$ challenge at $35^{\circ} \mathrm{C}$, the $96 \mathrm{peg}$ lids were removed and washed three times with sterile PBS, then placed onto new 96well microtiter plates containing $180 \mu \mathrm{l}$ recovery broth (MHB) and incubated for a further $24 \mathrm{~h}$ at $35{ }^{\circ} \mathrm{C}$. After the recovery incubation, the 96 peg lids were washed three times with sterile PBS, then placed onto new 96-well microtiter plates containing $200 \mu \mathrm{l}$ of reaction solution $(190 \mu \mathrm{l}$ MHB and $10 \mu \mathrm{l}$ detection reagent). After incubation at $35^{\circ} \mathrm{C}$, the generated formazan was measured at 460 nm with a microplate reader (VersaMax, Molecular Devices Co., Sunnyvale, CA USA). The MBEC was read as the lowest concentration of antibiotics for which

(1)

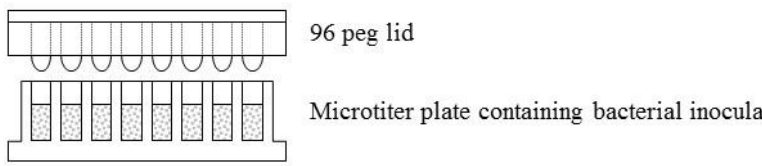

(2)
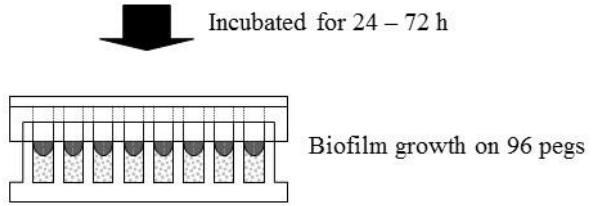

Washed the biofilm formed on 96 pegs three times with microtiter plate containing PBS

(3)

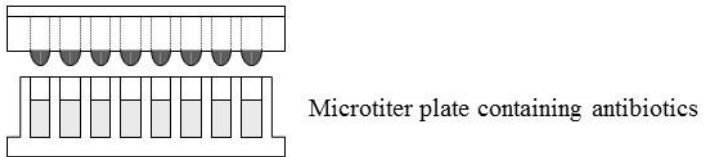

Challenged for $20 \mathrm{~h}$

(4)

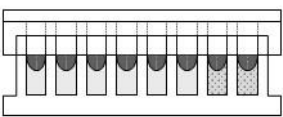

Washed the biofilm formed on 96 pegs three times with microtiter plate containing PBS the absorbance change (relative to the blank value without bacteria) was less than 0.1 .

Reference MBECs are determined according to the conventional method reported by Harrison et al. (Harrison et al., 2005, 2010). The conventional method computes the MBECs from the number of bacteria in the established biofilms. After the recovery incubation described above, the 96 peg lids are placed in another 96-well microtiter plates containing growth recovery broth, and then sonicated in an ultrasonic sonicating bath. Viable cells in the biofilm can be detected by plating onto appropriate medium, or by measuring increased turbidity over time, using a microplate reader. However, some of viable cells in the biofilm established on the 96 peg lids might be left behind for lack of sufficient sonication. Thus, to implement the conventional method, we collected the biofilm matrix by carefully swabbing each peg with a sterile cotton swab. The tip of the cotton swab was then inserted into a tube containing sterile PBS. The tube was vortexed and sonicated to disintegrate the bacterial clumps and to dislodge the bacteria from the tip of the cotton swab. The bacterial suspension was plated onto agar medium and the number of viable bacteria were counted after $24 \mathrm{~h}$ incubation. The MBEC was taken as the minimum concentration of antibiotics for which no visible colonies appeared.

\section{RESULTS AND DISCUSSION}

\section{Biofilm formation on the 96-pegs}

To establish the biofilm on the 96 pegs, we first noted the optimal growth conditions of $S$. aureus and $P$. aeruginosa, such as peg materials, growth medium, and incubation time. $S$. aureus more readily formed biofilms on polystyrene pegs than on polypropylene (data not shown). On the other hand, $P$. aeruginosa established biofilms more effectively on polypropylene pegs. The biofilm formation medium was MHB because this medium is most commonly used in susceptibility testing. P. aeruginosa formed biofilms on the 96 pegs within $24 \mathrm{~h}, \mathrm{~S}$. aureus required $72 \mathrm{~h}$. The biofilms established on the 96 pegs under the optimum conditions were tested for the determination of the MBECs as shown in Fig. 1.

(5)

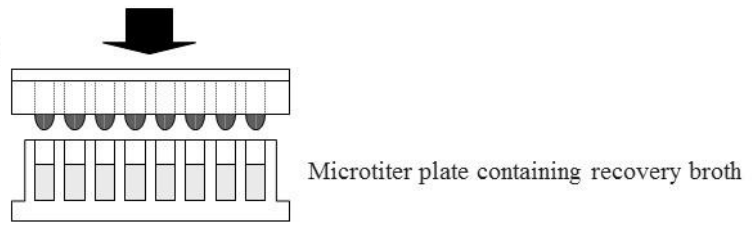

(6)
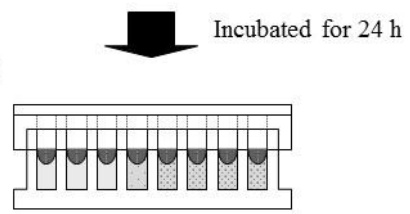

(7)

Washed the biofilm formed on 96 pegs three times with microtiter plate containing PBS

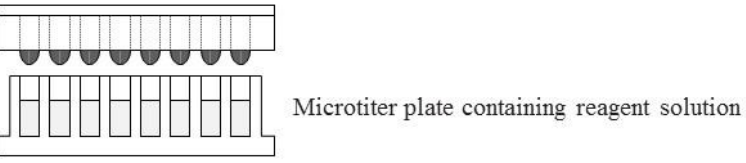

(8)

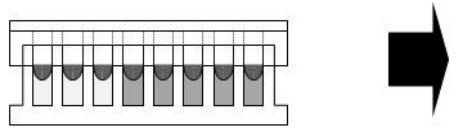

Measured at $460 \mathrm{~nm}$

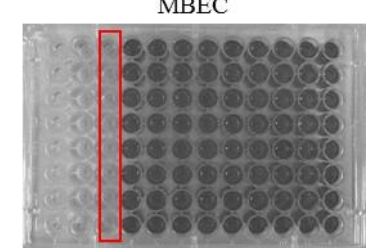

Figure 1 Procedures of biofilm susceptibility tests

\section{Susceptibility of biofilms exposed to a single antibiotic}

To evaluate the applicability of the WST-8 colorimetric method to rapid determination of the MBEC, we determined the biofilm susceptibility of $S$ aureus and $P$. aeruginosa in the presence of various single antibiotics by the proposed method.
Figure 2 shows the effects of the WST- 8 reduction time on the susceptibility curves of $S$. aureus NBRC13276 and $P$. aeruginosa NBRC13275. For $S$. aureus exposed to vancomycin, the MBEC was estimated as $512 \mu \mathrm{g} / \mathrm{ml}$ at a WST-8 reduction time of $2 \mathrm{~h}$ (Fig. 2 (A)). The MBECs obtained at $2 \mathrm{~h}$ and $4 \mathrm{~h}$ were almost equal. The MBEC for $P$. aeruginosa exposed to ciprofloxacin was estimated as $256 \mu \mathrm{g} / \mathrm{ml}$ at $4 \mathrm{~h}$, but the absorbance at $2 \mathrm{~h}$ was relatively low (Fig. 2 
(B)). From these results, the WST-8 reduction time was decided as $2 \mathrm{~h}$ for $S$. aureus and $4 \mathrm{~h}$ for $P$. aeruginosa.
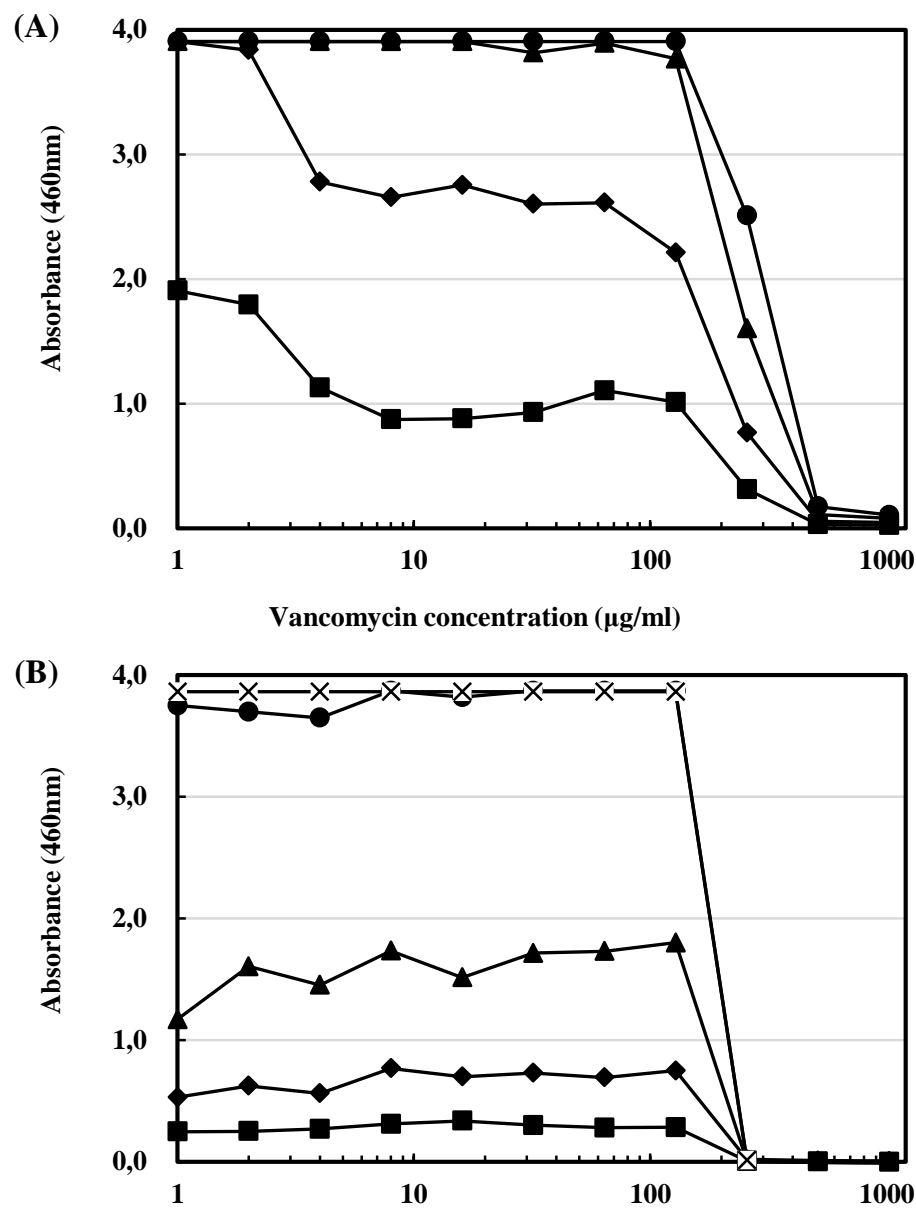

Ciprofloxacin concentration $(\mu \mathrm{g} / \mathrm{ml})$

Figure 2 Absorbance (used to determine the MBEC) versus antibiotic concentration for different reduction times of WST-8. Biofilms of S. aureus NBRC13276 (A) and P. aeruginosa NBRC13275 (B).

Incubation time: $1 \mathrm{~h}, \boldsymbol{\square} ; 2 \mathrm{~h}, \bullet ; 3 \mathrm{~h}, \boldsymbol{\Delta} ; 4 \mathrm{~h}, \boldsymbol{\bullet} ; 5 \mathrm{~h}, \times$.

The data represent the means for 4 identical wells of a microtiter plate from each experiment.

Tables 1 and 2 show the MBECs of S. aureus NBRC13276 and P. aeruginosa NBRC13275, respectively, exposed to various antibiotics and evaluated by the proposed and conventional methods. The most effective inhibitors of $S$. aureus and $P$. aeruginosa biofilms were vancomycin and ciprofloxacin, respectively. In addition, reference MBECs were also determined by the conventional method. There was good agreement between the MBECs obtained at 2-4 h using the WST-8 colorimetric method and those obtained after $24 \mathrm{~h}$ using the conventional method. In both $S$. aureus and $P$. aeruginosa biofilms, the percentage of the MBEC values located at $\pm 1 \log _{2}$ difference was $100 \%$. To better assess the degree of agreement between the MBEC results obtained from the present method and the conventional method, the Wilcoxon signed-ranked test was performed. $\mathrm{P}$ values derived from the Wilcoxon signed-rank test demonstrated no significant differences $(P=0.317)$ between the proposed method and the conventional method.

The conventional method is complicated and the MBEC assays are time consuming because the microbial cells must be first extracted from the biofilm matrix and then incubated for another $24 \mathrm{~h}$ (Harrison et al., 2005, 2010). On the other hand, the proposed method is simple to implement and yields the MBEC results within a few hours. In addition, the proposed method provides a fast assay of the MBEC. These findings indicate that the proposed method could potentially circumvent the tedious and time-consuming conventional method.
Table 1 MBECs of Staphylococcus aureus NBRC13276 biofilms exposed to single antibiotics

\begin{tabular}{lcc}
\hline Antibiotics & Proposed method & Conventional method \\
\hline Azithromycin & $1024<$ & $1024<$ \\
Cefazolin & $1024<$ & $1024<$ \\
Chloramphenicol & $1024<$ & $1024<$ \\
Ciprofloxacin & $1024<$ & $1024<$ \\
Clarithromycin & $1024<$ & $1024<$ \\
Clindamycin & $1024<$ & $1024<$ \\
Daptomycin & $1024<$ & $1024<$ \\
Doxycycline & 1024 & 1024 \\
Erythromycin & $1024<$ & $1024<$ \\
Gentamicin & $1024<$ & $1024<$ \\
Imipenem & $1024<$ & $1024<$ \\
Kanamycin & $1024<$ & $1024<$ \\
Linezolid & $1024<$ & $1024<$ \\
Meropenem & $1024<$ & $1024<$ \\
Minocycline & 1024 & 1024 \\
Oxacillin & $1024<$ & $1024<$ \\
Phosphomycin & $1024<$ & $1024<$ \\
Rifampicin & $1024<$ & $1024<$ \\
Streptomycin & $1024<$ & $1024<$ \\
Teicoplanin & $1024<$ & $1024<$ \\
Tigecycline & 1024 & $1024<$ \\
Tylosin & $1024<$ & $1024<$ \\
Vancomycin & 512 & 512 \\
\hline & & \\
& &
\end{tabular}

Table 2 MBECs of Pseudomonas aeruginosa NBRC13275 biofilms exposed to single antibiotics

\begin{tabular}{lcc}
\hline Antibiotics & Proposed method & Conventional method \\
\hline Amikacin & $1024<$ & $1024<$ \\
Azithromycin & $1024<$ & $1024<$ \\
Aztreonam & $1024<$ & $1024<$ \\
Ceftazidime & $1024<$ & $1024<$ \\
Chloramphenicol & $1024<$ & $1024<$ \\
Ciprofloxacin & 256 & 256 \\
Clarithromycin & $1024<$ & $1024<$ \\
Colistin & $1024<$ & $1024<$ \\
Doxycycline & $1024<$ & $1024<$ \\
Enrofloxacin & 1024 & 1024 \\
Gentamicin & $1024<$ & $1024<$ \\
Imipenem & $1024<$ & $1024<$ \\
Kanamycin & $1024<$ & $1024<$ \\
Levofloxacin & 1024 & 1024 \\
Meropenem & $1024<$ & $1024<$ \\
Ofloxacin & $1024<$ & $1024<$ \\
Polymyxin B & $1024<$ & $1024<$ \\
Streptomycin & $1024<$ & $1024<$ \\
Tobramycin & $1024<$ & $1024<$ \\
\hline
\end{tabular}

$(\mu \mathrm{g} / \mathrm{ml})$

\section{Susceptibility testing of biofilms exposed to plural antibiotics}

To investigate the combined effect of antibiotics against microbial biofilm, we applied the proposed method to the biofilm susceptibility of $S$. aureus exposed to combinations of plural antibiotics. Tables 3 and 4 present the MBECs of $S$. aureus in the presence of two and three antibiotics, respectively.

In the two-antibiotic combinations, each antibiotic was compared with vancomycin, the most effective inhibitor of $S$. aureus biofilm in the singleantibiotic study. Vancomycin combined with ciprofloxacin, daptomycin, and teicoplanin effectively reduced the MBEC (Table 3). Therefore, in the threeantibiotic combination, several antibiotics were combined with vancomycin and daptomycin. As shown in Table 4, vancomycin, daptomycin, and teicoplanin most effectively reduced the MBEC of $S$. aureus biofilm. The effectiveness of the vancomycin-daptomycin-teicoplanin combination was also investigated for other $S$. aureus strains. Clearly, biofilms established by other $S$. aureus, including methicillin-resistant $S$. aureus (MRSA), were largely inhibited by this drug combination. In the absence of teicoplanin, the combination of vancomycin and 
daptomycin hardly inhibited other $S$. aureus with the exception of $S$. aureus NBRC13276 (data not shown). Therefore, it is thought that the addition effect of teicoplanin is very important for $S$. aureus biofilm eradication.

The glycopeptide antibiotics, such as vancomycin and teicoplanin have similar mechanisms of action on bacterial cell wall synthesis. Vancomycin and teicoplanin are both widely used in the treatment of infections caused by Grampositive bacteria. Teicoplanin has a longer half-life than vancomycin (Murphy and Pinney, 1995; Wood, 1996). Thus, vancomycin requires multiple dosing to maintain adequate serum levels. As shown in Table 1, vancomycin alone showed higher biofilm eradication activity than teicoplanin. In the combination of vancomycin and teicoplanin, it is thought that vancomycin acts $S$. aureus biofilms potentially at an early stage, and then teicoplanin mainly affects the biofilms after the effectiveness of vancomycin is attenuated.

Saginur et al. (2006) reported that most of the effective antibiotic combinations against $S$. aureus (including MRSA) include rifampicin. Rifampicin is especially effective when combined with vancomycin and/or fusidic acid (Saginur $\boldsymbol{e t}$. $\boldsymbol{a l}$. 2006). Vancomycin is often combined with a secondary antibiotic, usually rifampicin or gentamicin, for the treatment of serious MRSA infections (Deresinski, 2009). We demonsrtrated that a new combination of vancomycin, daptomycin and teicoplanin was most effective against $S$. aureus biofilms in vitro.

In the last decade, the role of biofilms in various chronic bacterial infections has been recognized (Burmolle $\boldsymbol{e t}$ al., 2010). The findings of the present study suggest the utility of the proposed method as a rapid and simple assay of microbial biofilm. In the future, we hope that our method will be adopted as an alternative to the conventional method for rapid and simple susceptibility testing of microbial biofilm.

Table 3 MBECs of Staphylococcus aureus NBRC13276 biofilms exposed to combinations of two antibiotics, determined by the proposed method

\begin{tabular}{lcccc}
\hline \multirow{2}{*}{ Antibiotics } & \multicolumn{4}{c}{ Vancomycin } \\
\cline { 2 - 5 } & 16 & 32 & 64 & 128 \\
\hline Cefazolin & $128<$ & $128<$ & $128<$ & $128<$ \\
Ciprofloxacin & 128 & 128 & 128 & 64 \\
Clarithromycin & $128<$ & $128<$ & $128<$ & $128<$ \\
Clindamycin & $128<$ & $128<$ & $128<$ & $128<$ \\
Daptomycin & $128<$ & $128<$ & 64 & 4 \\
Gentamicin & $128<$ & $128<$ & $128<$ & 128 \\
Linezolid & $128<$ & $128<$ & $128<$ & $128<$ \\
Meropenem & $128<$ & $128<$ & $128<$ & $128<$ \\
Minocycline & $128<$ & $128<$ & $128<$ & $128<$ \\
Rifampicin & $128<$ & $128<$ & $128<$ & $128<$ \\
Teicoplanin & $128<$ & $128<$ & 2 & 1 \\
Tigecycline & $128<$ & $128<$ & $128<$ & $128<$ \\
\hline
\end{tabular}

$(\mu \mathrm{g} / \mathrm{ml})$

Table 4 MBECs of biofilms of various Staphylococcus aureus strains exposed to combinations of three antibiotics, determined by the proposed method

\begin{tabular}{lcccc}
\hline \multirow{2}{*}{ Antibiotics } & \multicolumn{4}{c}{ Vancomycin / Daptomycin } \\
\cline { 2 - 5 } & $8 / 4$ & $16 / 4$ & $32 / 4$ & $64 / 4$ \\
\hline S. aureus NBRC13276 & $32<$ & $32<$ & $32<$ & 8 \\
$\quad$ Ciprofloxacin & $32<$ & $32<$ & 32 & 2 \\
Gentamicin & $32<$ & $32<$ & $32<$ & $32<$ \\
Minocycline & $32<$ & $32<$ & $32<$ & 32 \\
Rifampicin & $32<$ & $32<$ & $32<$ & $32<$ \\
Tigecycline & 8 & 4 & 4 & 1 \\
Teicoplanin & $32<$ & $32<$ & $32<$ & 32 \\
S. aureus ATCC29213 & & & & \\
Teicoplanin & $32<$ & $32<$ & $32<$ & 32 \\
S. aureus NBRC14462 & $32<$ & $32<$ & 32 & 16 \\
Teicoplanin & & & & $(\mu \mathrm{g} / \mathrm{ml})$ \\
S. aureus ATCC43300 (MRS) & & & \\
$\quad$ Teicoplanin & & & & \\
\hline &
\end{tabular}

\section{CONCLUSION}

We have develop the rapid and simple method for the determination of MBEC using a colorimetric microbial viability assay based on reduction of a tetrazolium salt WST-8 and the biofilm formation method on 96 pegs on the lid of a microtiter plate, and demonstrated the advantages of the proposed method as compared to the conventional methods. There was good agreement between the MBECs obtained at 2-4 h using the WST-8 colorimetric method and those obtained after $24 \mathrm{~h}$ using the conventional method. In addition, by the proposed method, we clarified that a combination of vancomycin, daptomycin and teicoplanin was most effective for Staphylococcus aureus biofilm. The proposed method yields similar performance to conventional methods, but is faster and more easily implemented. Therefore, the proposed method alleviates the tediousness and time-consuming nature of conventional biofilm susceptibility assay.

Acknowledgments: This work was supported by a Japan Society for the Promotion of Science KAKENHI Grant No. 25450123.

\section{REFERENCES}

Aaron, S. D., Ferris, W., Ramotar, K., Vandemheen, K., Chan, F. \& Saginur, R (2002) Single and combination antibiotic susceptibilities of planktonic, adherent, and biofilm-grown Pseudomonas aeruginosa isolates cultured from sputa of adults with cystic fibrosis. J. Clin. Microbiol., 40, 4172-4179. http://dx.doi.org/10.1128/JCM.40.11.4172-4179.2002

Antunes, A. L. S., Trentin, D. S., Bonfanti, J. W., Pinto, C. C. F., Perez, L. R. R. Macedo, A. J. \& Barth, A. L. (2010) Application of a feasible method for determination of biofilm antimicrobial susceptibility in staphylococci. APMIS, 118, 873-877. http://dx.doi.org/10.1111/j.1600-0463.2010.02681.x

Bhinu, V. S. (2005) Insight into biofilm-associated microbial life. J. Mol Microbiol. Biotechnol., 10, 15-21. http://dx.doi.org/10.1159/000090344

Bridiera, A., Briandeta, R., Thomasc, V. \& Dubois-Brissonneta, F. (2011) Resistance of bacterial biofilms to disinfectants: a review. Biofouling., 27, 1017 1032. http://dx.doi.org/ 10.1080/08927014.2011.626899

Burmølle, M., Thomsen, T. R., Fazli, M., Dige, I., Christensen, L., Homøe, P., Tvede, M., Nyvad, B., Tolker-Nielsen, T., Givskov, M., Moser, C., KirketerpMøller, K., Johansen, H. K., Høiby, N., Jensen, P. Ø., Sørensen, S. J. \& Bjarnsholt, T. (2010) Biofilms in chronic infections - a matter of opportunity monospecies biofilms in multispecies infections. FEMS Immunol. Med. Microbiol., 59, 324-336. http://dx.doi.org/10.1111/j.1574-695X.2010.00714.X Ceri, H., Olson, M., Morck, D., Storey, D., Read, R., Buret, A. \& Olson, B. (2001) The MBEC Assay System: multiple equivalent biofilms for antibiotic and biocide susceptibility testing. Methods Enzymol., 337, 377-385. http://dx.doi.org/10.1016/S0076-6879(01)37026-X

Deresinski, S. (2009) Vancomycin in combination with other antibiotics for the treatment of serious methicillin-resistant Staphylococcus aureus infections. Clin. Infect. Dis., 49, 1072-1079. http://dx.doi.org/10.1086/605572

Harrison, J. J., Turner, R. J., \& Ceri, H. (2005) High-throughput metal susceptibility testing of microbial biofilms. BMC Microbiology, 5, 53. http://dx.doi.org/10.1186/1471-2180-5-53

Harrison, J. J., Stremick, C. A., Turner, R. J., Allan, N. D., Olson, M. E. \& Ceri, H. (2010) Microtiter susceptibility testing of microbes growing on peg lids: a miniaturized biofilm model for high-throughput screening. Nature Protocols, 5, 1236-1254. http://dx.doi.org/10.1038/nprot.2010.71

Murphy, S., and Pinney, R. J. (1995) Teicoplanin or vancomycin in the treatment of Gram-positive infections? J. Clin. Pharm. Ther., 20, 5-11. http://dx.doi.org/10.1111/j.1365-2710.1995.tb00619.x

Nett, J. E., Cain, M. T., Crawford, K., and Andes, D. R. (2011) Optimizing a Candida biofilm microtiter plate model for measurement of antifungal susceptibility by tetrazolium salt assay. J. Clin. Microbiol., 49, 1426-1433. http://dx.doi.org/10.1128/JCM.02273-10

Qu, Y., Daley, A. J., Istivan, T. S., Rouch, D. A. \& Deighton, M. A. (2010) Densely adherent growth mode, rather than extracellular polymer substance matrix build-up ability, contributes to high resistance of Staphylococcus epidermidis biofilms to antibiotics-authors' response. J. Antimicrob. Chemother., 65, 1405-1411. http://dx.doi.org/10.1093/jac/dkq275

Saginur, R., Denis, M. S., Ferris, W., Aaron, S. D., Chan, F., Lee, C. \& Ramotar, K. (2006) Multiple combination bactericidal testing of staphylococcal biofilms from implant-associated infections. Antimicrob. Agents Chemother., 50, 55-61. http://dx.doi.org/10.1128/AAC.50.1.55-61.2006

Tsukatani, T., Suenaga, H., Higuchi, T., Akao, T., Ishiyama, M., Ezoe, T. \& Matsumoto, K. (2009) Colorimetric microbial viability assay based on reduction of water-soluble tetrazolium salts for antimicrobial susceptibility testing and screening of antimicrobial substances. Anal. Biochem., 393, 117-125. http://dx.doi.org/10.1016/j.ab.2009.06.026

Tsukatani, T., Suenaga, H., Shiga, M., Noguchi, K., Ishiyama, M., Ezoe, T. \& Matsumoto, K. (2012) Comparison of the WST-8 colorimetric method and the CLSI broth microdilution method for susceptibility testing against drug-resistant bacteria. J. Microbiol. Methods, 90, 160-166. http://dx.doi.org/10.1016/j.mimet.2012.05.001

Wood, M. J. (1996) The comparative efficacy and safety of teicoplanin and vancomycin. J. Antimicrob. Chemother., 37, 209-222. http://dx.doi.org/ 10.1093/jac/37.2.209

Zhang, L. \& Mah, T. F. (2008) Involvement of a novel efflux system in biofilmspecific resistance to antibiotics. J. Bacteriol., 190, 4447-4452. http://dx.doi.org/ $\underline{10.1128 / \mathrm{JB} .01655-07}$ 